\title{
Molecular docking analysis of UniProtKB nitrate reductase enzyme with known natural flavonoids
}

\author{
Ayub Shaik1, Vishnu Thumma², Aruna Kumari Kotha², Sandhya Kramadhati², Jalapathy \\ Pochampally², Seshagiri Bandi ${ }^{*}$
}

${ }^{1}$ Department of Chemistry, University College of Science, Osmania University, Hyderabad, Telangana, India; ${ }^{2}$ Department of Sciences and Humanities, Matrusri Engineering College, Hyderabad, Telangana, India; ${ }^{3 B}$ Bioinformatics Division, Osmania University, Hyderabad, Telangana, India; Seshagiri Bandi - E-mail: giri.bandi@gmail.com

Received October 21, 2016; Accepted November 11, 2016; Published December 27, 2016

\begin{abstract}
:
The functional inference of UniProtKB nitrate reductase enzyme (UniProtKB - P0AF33) through structural modeling is of interest in plant biology. Therefore, a homology model for UniProtKB variant of the enzyme was constructed using available data with the MODELER software tool. The model was further docked with five natural flavonoid structures such as hesperetin, naringenin, leucocyanidin, quercetin and hesperetin triacetate using the AUTODOCK (version 4.2) software tool. The structure aided molecular interactions of these flavonoids with nitrate reductase is documented in this study. The binding features (binding energy $(\Delta \mathrm{G})$ value, $\mathrm{H}$ bonds and docking score) hesperetin to the enzyme model is relatively high, satisfactory and notable. This data provides valuable insights to the relative binding of several naturally occurring flavonoids to nitrate reductase enzyme and its relevance in plant biology.
\end{abstract}

Keywords: Homology modeling, nitrate reductase, natural flavonoids, docking

\section{Background:}

Nitrogen is one of the most important growth-limiting nutrients in plants. The major source of nitrogen in most of the higher plants is nitrate $\left(\mathrm{NO}_{3}\right)$ absorbed through roots. Nitrate can be reduced both in the photosynthetic tissues and in non-photosynthetic tissues such as roots [1]. Nitrate reductase catalyses the oxidation of $\mathrm{NAD}(\mathrm{P}) \mathrm{H}$ and the reduction of nitrate to nitrite [2]. This is subject to control at the levels of enzyme activity, synthesis, and degradation [3]. Nitrate reductase catalyzes the reduction of nitrate via nitrite to ammonia for the anabolic incorporation of nitrogen into bio-molecules [4]. Nitrate reduction can be performed with different purposes (a) nitrate assimilation: the utilization of nitrate as a nitrogen source for growth, (b) nitrate respiration: the generation of metabolic energy by using nitrate as a terminal electron acceptor (c) nitrate dissimilation: the dissipation of excess reducing power for redox balancing [5]. Thus, the importance of nitrate reductase in nitrogen fixation is known. The interaction of naturally occurring flavonoids to the enzyme is of significance in

ISSN 0973-2063 (online) 0973-8894 (print) plant biology. Therefore, it is of interest to document the molecular docking based interaction analysis of nitrate reductase enzyme with known natural flavonoids.

\section{Methodology:}

Sequence data:

Protein sequence (226 residues) of UniProtKB - P0AF33 nitrate reductase was retrieved from Uniprot [6].

\section{Template search:}

A sequence similarity search was performed using the Protein BLAST [7] tool to identify the structural template from Protein Data Bank (PDB) for homology modeling [8]. The entry with PDB ID: 1 Q16 having an identity of $72 \%$ with UniProtKB - P0AF33 was selected as a template for homology modeling. 


\section{BIOINFORMATION \\ Discovery at the interface of physical and biological sciences}

\section{Open access}

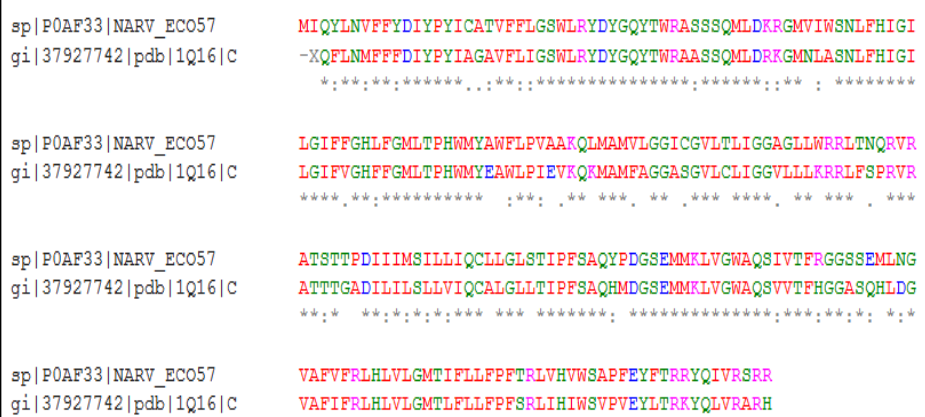

MIQYLNVFFYDIYPYICATVFFLGSWLRYDYGQYTWRASSSQMLDKRGMVIWSNLFHIGI -XQFLNMFFFIYPYIAGAVELIGSWLRYDYGQYTWRAASSQMLDRRGMNLASNLFHIGI

IGIFFGHLFGMLTPHWMYAWELPVAARQIMAMVLGGICGVLTLIGGAGLLWRRLTNQRVR LGIFVGHFFGMLTPHWMYEAWLPIEVRQRMAMFAGGASGVLCLIGGVLILRRRLFSPRVR

ATSTTPDIIIMSILLIQCLLGLSTIPFSAQYPDGSEMMKLVGWAQSIVTERGGSSEMLNG ATITGADILILSLIVIQCALGLITIPFSAQHMDGSEMMRLVGWAQSVVTFHGGASQHLD

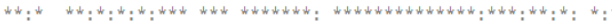

VAFVFRLHLVIGMTIFLLFPFTRLVHVWSAPFEYFTRRYQIVRSRB VAFIFRLHLVIGMTLFLLFPFSRLIHIWSVPVEYLTRKYQLVRARH

Figure 1: Sequence alignment of UniProtKB nitrate reductase with the known template structure (PDB ID: 1Q16) having $72 \%$ of identity and $84 \%$ similarity.

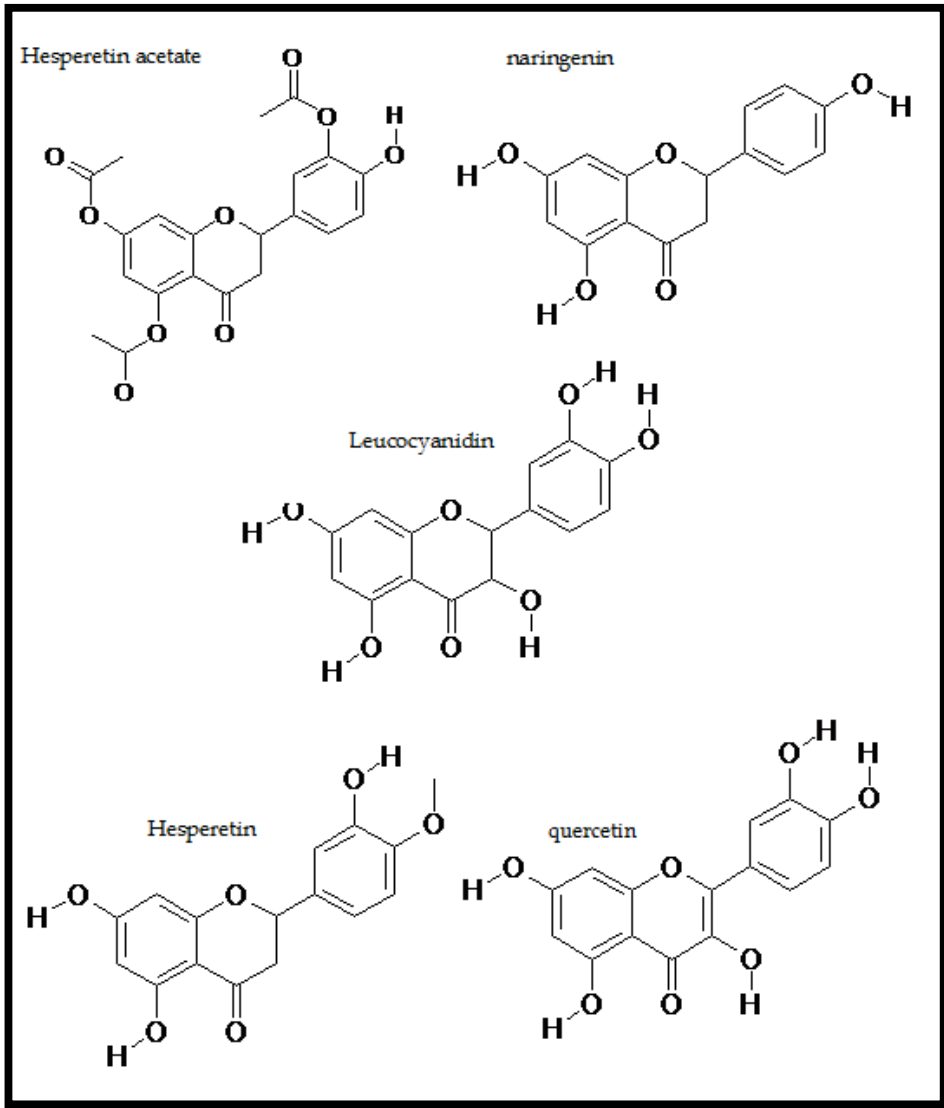

Figure 2: 2D structures of natural flavonoids used for docking is shown

Sequence alignment:

The online ClustalW tool [9] was used for sequence alignment. Figure 1 shows the sequence alignment of UniProtKB - P0AF33 and template.

Homology modeling:

A homology model for UniProtKB - P0AF33 was subsequently generated using MODELLER version 9.16 [10]. The generated model was further checked for structure stereo-chemistry including Ramachandran plot and Psi/Phi angles using PROCHECK [11]

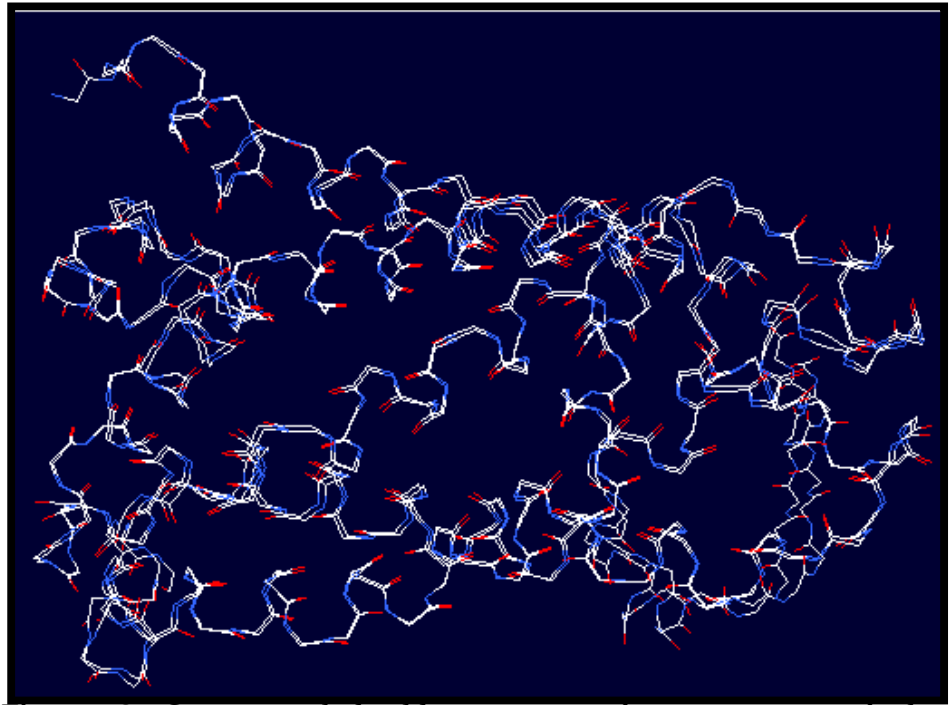

Figure 3: Superposed backbone traces for structures of the UniProtKB model and template (PDB ID: 1Q16). The structures were superimposed using SWISS PDB viewer (spdbv).

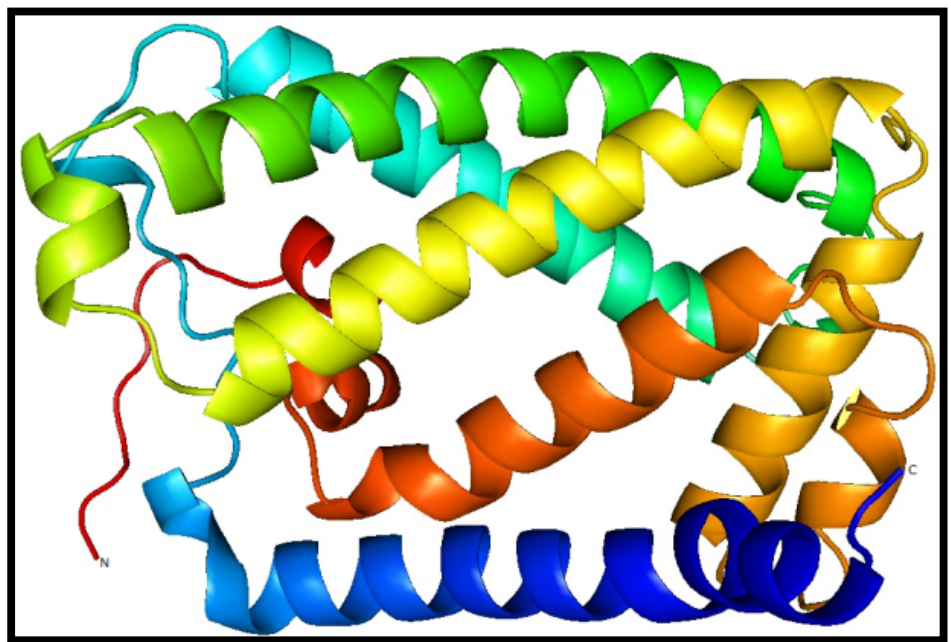

Figure 4: Cartoon representation of UniProtKB - P0AF33 nitrate reductase model with marked $\mathrm{C}$ terminal and $\mathrm{N}$ terminals. 


\section{BIOINFORMATION \\ Discovery at the interface of physical and biological sciences}

\section{Open access}

\section{Ligand structures:}

The structures of Musa paradiasica (common name: banana) extracted flavonoids Hesperetin (IUPAC Name: 5,7-Dihydroxy-2(3-hydroxy-4-methoxy-phenyl)-chroman-4-one), Naringenin (IUPAC Name: 5,7-Dihydroxy-2-(4-hydroxy-phenyl)-chroman-4one), Leucocyanidin (IUPAC Name: 2-(3,4-Dihydroxy-phenyl)3,5,7-trihydroxy-chroman-4-one), Quercetin (IUPAC Name: 2-(3,4Dihydroxy-phenyl)-3,5,7-trihydroxy-chromen-4-one), Hesperetin triacetate (IUPAC Name: Acetic acid 5-[7-acetoxy-5-(1-hydroxyethoxy)-4-oxo-chroman-2-yl]-2-hydroxy-phenyl ester) were shown (Figure 2). The ligand structures were sketched in SYBYL version 6.7 [12] and subsequently energy minimized. The structures were then saved in .mol2 format for further analysis.

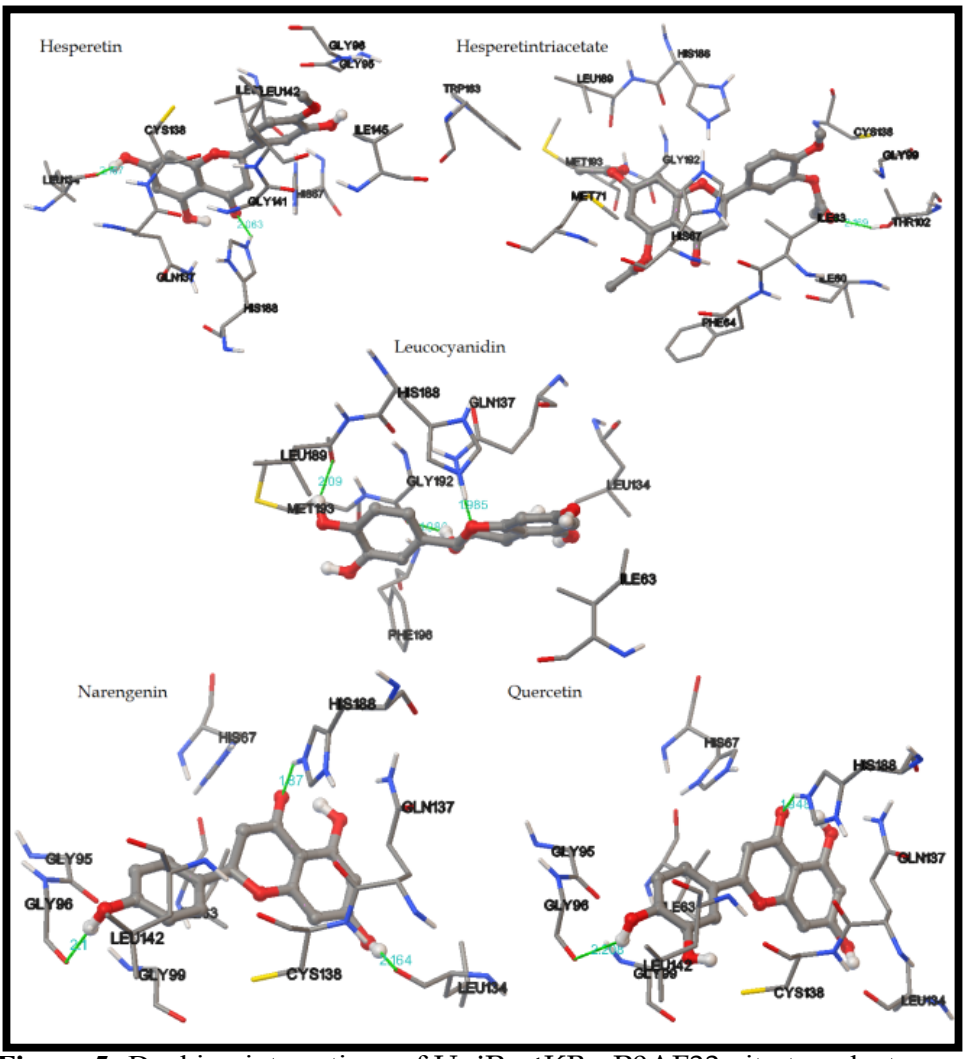

Figure 5: Docking interactions of UniProtKB - P0AF33 nitrate reductase model with known flavonoids such as hesperetin, hesperetintriacetate, leucocyanidin, narengenin and quercetin.

\section{Molecular docking:}

Molecular docking studies were performed using the Autodock (version 4.2) software tool [13]. The structures were optimized by adding hydrogens using kollaman charges [14]. The model were prepared by optimizing torsion angles and saved in PDBQT format. Potential binding site for the nitrate reductase protein was identified using 3Dligand site [15]. A grid was generated to identify

ISSN 0973-2063 (online) 0973-8894 (print) xyz coordinates $(X=-149.455, Y=-6.672$ and $Z=-16.321)$ around the binding site of the enzyme. Lamarckian genetic algorithm (LGA) was selected for freezing, docking with default parameters in Autodock.

Accessible surface area (ASA) versus residue number plot: ASA plot of nitrate reductase was completed using ASA-View, a database and tools for the solvent accessibility representation in proteins [17]. A characteristic 2D spiral plot of solvent accessibility provides a convenient graphical view of residues in terms of their exposed surface areas (Figure 6).

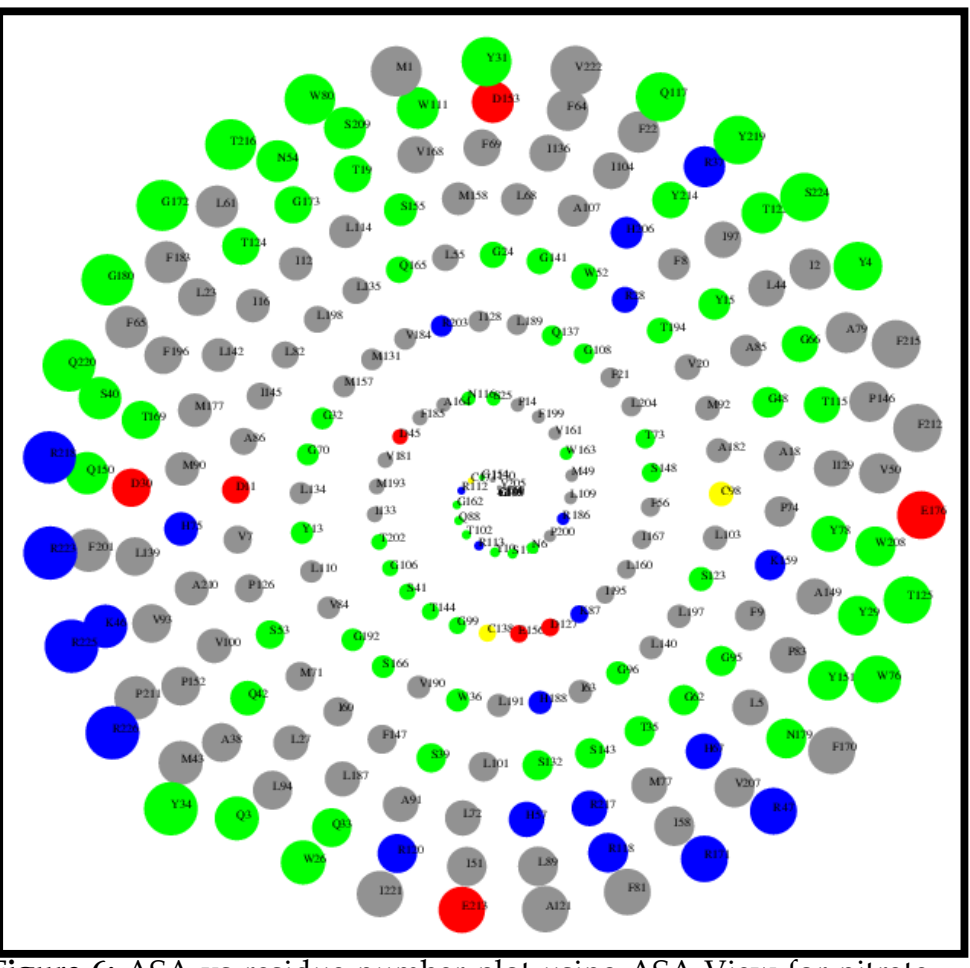

Figure 6: ASA vs residue number plot using ASA-View for nitrate reductase. The colors are coded as Blue for Positive charged residues $(R, K, H)$, Red for Negative charged residue (D, E), Green for Polar uncharged residues $(G, N, Y, Q, S, T, W)$, Yellow for Cystein and Gray for Hydrophobic residues (all others) for model.

Electrostatic distribution of the modeled surface:

The electrostatic potential distribution of the nitrate reductase enzyme model was analyzed using UCSF Chimera (a highly extensible tool for the analysis of molecular structure) [16]. Electrostatic surface mapping of nitrate reductase was completed for distribution and charge related properties of the enzyme model. The surface of nitrate reductase was color coded as per the Coulomb's law (Figure 7). 


\section{Open access}

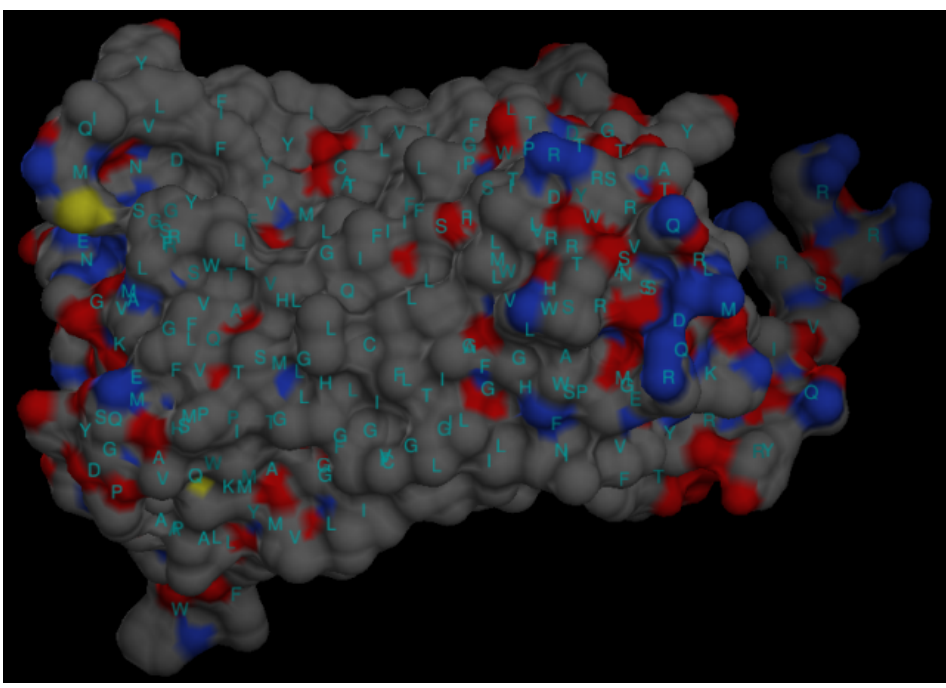

Figure 7: Electrostatic surface distribution of the modeled surface produced using UCSF Chimera. The surface was color coded as per the standard protocol of UCSF Chimera. Each amino acid was marked with standard code (blue for positive potential, white for neutral potential and red for negative potential).

\section{Results and Discussion:}

A molecular model (Figure 4) of UniProtKB nitrate reductase enzyme (UniProtKB - P0AF33) was constructed using homology (Figure 3) modeling techniques (with crystal structure of nitrate reductase A, NarGHI, from Escherichia coli (PDB entry: 1Q16) as template structure (Figure 1)) in MODELER version 9.16 and validated as described in the methodology section. The solvent accessible surface area (Figure 6) and the surface electrostatic distribution (Figure 7) of the enzyme model are presented. This information provides valuable insights to the physical and chemical features of the enzyme model towards its functional inference.

The model was further used for the structural docking of five banana derived natural flavonoids (Figure 2). The molecular interactions of the five flavonoids with the nitrate reductase model are shown in Figure 5. The characteristics binding (binding energy $(\Delta \mathrm{G})$ value, $\mathrm{H}$ bonds and docking score) of flavonoids to the reductase enzyme is given in Table 1 (see page 429). Among the five $M$. paradiasica derived secondary metabolites of hesperitin triacetate, naringenin, quercetin and hesperitin showed binding energy $(\Delta G)$ values of $-8.36,-7.53,-7.32$ and $-6.72 \mathrm{kcal}$ per mole, respectively. The compound leucocyanidin shows the least binding energy of -5.76 with three hydrogen-bonding interactions with Q137, G192 and L189.

Data shows the binding of hesperitin triacetate with the nitrate reductase protein model with notable features. Hesperitin triacetate shows a high binding energy of $-8.36 \mathrm{kcal}$ per mol and interacting with the residue T102 at a distance of $2.169 \AA$ A. A hydrogen bond was seen between hydrogen of T102 and the oxygen of hesperitin triacetate. Data shows that naringenin interacts with three amino acid residues H188, L134 and G96 with a docking score of $-7.53 \mathrm{kcal}$ per mol. It is also observed that quercetin shows a binding energy of $-7.32 \mathrm{kcal}$ per mol while interacting with H188 and G96.

\section{Conclusion:}

The binding characteristics of natural flavonoids such as hesperetin, naringenin, leucocyanidin, quercetin and hesperetin triacetate with the UniProtKB - P0AF33 structural model of nitrate reductase are documented in this study. The exercise shows that hesperitin triacetate having best binding features with the nitrate reductase protein model. This provides valuable insights towards the binding of natural flavonoids with the nitrate reductase enzyme and its importance in plant biology.

\section{References:}

[1] Karim Kherraz et al. Bioinformation. 2011 6(3): 115-119.

[2] Seenivasagan R. et al. Journal of Taibah University for Science 2016 10: 621-630.

[3] Fernández E et al. Proc Natl Acad Sci U S A. 1989 86(17): 64496453.

[4] Nakano MM et al. J Bacteriol. 1998 180(20): 5344-5350.

[5] Moreno-Vivián C et al. J Bacteriol. 1999 181(21):6573-84.

[6] http://www.uniprot.org/uniprot/P0AF33

[7] Camacho C et al. BMC Bioinformatics 2009 10:421

[8] http://www.rcsb.org/pdb/home/home.do

[9] Larkin MA et al. Bioinformatics 2007 23(21): 2947-2948.

[10] Andrej Sali et al. J.Mol.Biol. 1993 234: 779-815.

[11] Fischmann TO et al. Nat Struct Biol. 1999 6: 233 [PMID: 10074942]

[12] Gunda et al. Int J Comput Biol Drug Des. 2015 8(1): 19-39

[13] Morris GM et al. J. Computational Chemistry 2009 16: 2785-2791.

[14] Rashidieh et al. Bioinformation. 2015 11: 501 [PMID: 26912950]

[15] Wass MN et al. NAR 2010 38: Suppl: W469-73.

[16] Pettersen EF et al. J Comput Chem. 2004 25(13): 1605-12 [PMID:15264254]

[17] Ahmad S et al. BMC Bioinformatics. 2004 5: 51.[PMID: 15119964]

Edited by $P$ Kangueane

Citation: Shaik et al. Bioinformation 12(12): 425-429 (2016) License statement: This is an Open Access article which permits unrestricted use, distribution, and reproduction in any medium, provided the original work is properly credited. This is distributed under the terms of the Creative Commons Attribution License
BIOMEDICAL

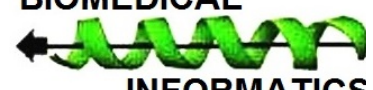




\section{BIOINFORMATION}

Discovery at the interface of physical and biological sciences

\section{Open access}

Table 1: Hydrogen bond interactions of flavonoids with the nitrate reductase model

\begin{tabular}{lllll}
\hline Ligand & Interacting amino acids & Grid X-Y-Z coordinates & Binding energy $\mathbf{\Delta G}($ Kcal/Mol) & Dissociation constant (kl) $(\boldsymbol{\mu M})$ \\
\hline Hesperetin acetate & Thr102 & $-149.455,-6.672-16.321$ & -8.36 & 749.04 \\
Naringenin & His188, Leu135, Gly96 & $-149.455,-6.672-16.321$ & -7.53 & 3.02 \\
Leucocyanidin & Gln137, Leu189, Gly192 & $-149.455,-6.672-16.321$ & -5.76 & 60.37 \\
Quercetin & His188, Gly96 & $-149.455,-6.672-16.321$ & -7.32 & 4.34 \\
Hesperetin & His188, Leu134 & $-149.455,-6.672-16.321$ & -6.72 & 11.95 \\
\hline
\end{tabular}

